\title{
Improved matrix coating for positive- and negative-ion-mode MALDI-TOF imaging of lipids in blood vessel tissues
}

\author{
Christina Meisenbichler ${ }^{1} \cdot$ Christian Doppler $^{2} \cdot$ David Bernhard $^{2} \cdot$ Thomas Müller $^{1}$ \\ Received: 13 February 2019 / Revised: 25 March 2019 / Accepted: 3 April 2019 / Published online: 30 April 2019 \\ (C) The Author(s) 2019
}

\begin{abstract}
High-quality matrix-assisted laser desorption/ionization mass spectrometry imaging (MALDI MSI) of lipids in biological tissue relies on the fabrication of a homogeneous matrix coating featuring best possible analyte integration. This communication addresses a matrix vapor deposition/recrystallization process for the application of 1,5-diaminonaphthalene (1,5-DAN) onto slices of human aortic tissue. The matrix coating is compatible with both positive- as well as negative-ion-mode MALDI MSI facilitating a significantly enhanced detection of lipid-related signals in different cell layers of blood vessel walls.
\end{abstract}

Keywords MALDI MSI $\cdot$ Matrix vapor deposition/recrystallization $\cdot$ Lipidomics $\cdot$ Thoracic aortic aneurysm

\section{Introduction}

Mass spectrometry imaging (MSI) has become a powerful tool for the analysis of biological tissue, in particular due to its potential to monitor 2D distributions of chemicals in different tissue layers [1-3]. In matrix-assisted laser desorption/ ionization mass spectrometry imaging (MALDI MSI), the fabrication of a homogeneous, analyte containing matrix coating [4] is a prerequisite for high sensitivity but also the key step for the acquisition of highly resolved data. This can be achieved by either applying a matrix in solution [5-10] or depositing solvent-free matrix onto the sample surface [11-15]. Although the application of a dissolved matrix (usually using an automated spray system [16]) features an immediate orthogonal integration of the analytes into the matrix, it involves the risk of lateral analyte delocalization, which is a clear limitation for high-resolution imaging. On the other side,

Electronic supplementary material The online version of this article (https://doi.org/10.1007/s00216-019-01826-x) contains supplementary material, which is available to authorized users.

Thomas Müller

thomas.mueller@uibk.ac.at

1 Institute of Organic Chemistry, Leopold-Franzens University Innsbruck, 6020 Innsbruck, Austria

2 Center for Medical Research, Medical Faculty, Johannes Kepler University Linz, 4020 Linz, Austria matrix vapor deposition [11] produces exceptionally homogeneous coatings and precludes artifacts due to analyte diffusion [17]. Lacking integration of analytes into coating can easily be compensated by recrystallization of the matrix layer $[18,19]$. Moreover, sublimation of the matrix is an additional purification step resulting in excellent low chemical noise within the MALDI mass spectrum.

Thoracic aortic aneurysms (TAA) are among the 20 most relevant causes of death in all individuals and the 17th most common in elderly patients above 65 years [20]. An exact number of cases is difficult to determine, since most aortic aneurysms are clinically silent and only around 5\% of all patients are symptomatic before an acute event occurs [21]. A previous metabolome-based study provided first evidence that lipids might also be potential biomarkers for the occurrence of thoracic aortic aneurysms (TAA) [22]. In a targeted metabolomics approach, tissue concentrations of sphingomyelins and phosphatidylcholines were identified to potentially distinguish pathological from healthy tissue. In order to strengthen the significance of these findings, we utilized MSI to both localize lipids in different tissue layers as well as overcome the limits of a targeted analysis focusing on a restricted number of possible biomarker candidates.

In this study, we present a concise sample preparation method for MALDI MSI to examine the histological distribution of lipids in aortic tissue based on the matrix deposition/ recrystallization methodology. Method development was focused on (i) a short sample preparation procedure, (ii) a possibility of keeping the sample cooled or under vacuum as long 
as possible, and (iii) enhancing sensitivity for the identification of a maximum number of lipid-related signals.

\section{Material and methods}

\section{Reagents and chemicals}

1,5-Diaminonaphthalene (1,5-DAN) was purchased from Sigma-Aldrich (St. Louis, USA). Isopropanol, methanol, toluene, and chloroform were purchased from VWR (Fontenay Sous Bois, France), and acetonitrile and ammonium formate from Riedel-de Haen (Seelze, Germany). Formic acid was purchased from Fluka (Buchs, Switzerland) and 0.9\% saline water from Fresenius Kabi (Graz, Austria).

\section{Tissue sections}

Frozen human aortic tissue was obtained from the Medical University of Innsbruck [22]. The human aortic tissue was stored at $-80{ }^{\circ} \mathrm{C}$ until it was cut into $8-\mu \mathrm{m}$ thick slices at $-20^{\circ} \mathrm{C}$ by using a Leica CM 1850 cryostat (Leica Microsystems GmbH, Wetzlar, Germany). The tissue sections were thaw-mounted on $1 \times 1 \mathrm{~cm}$ stainless steel or standard ITO slides (Bruker Daltonics) and stored again at $-80{ }^{\circ} \mathrm{C}$ until needed.

\section{Tissue washing step (optional)}

The thaw-mounted tissue sections were covered with 6 drops of saline water or ammonium formate solution (prepared according to [23]). After $30 \mathrm{~s}$ (or $1.5 \mathrm{~min}$ in case of ammonium formate), the washing solution was removed by tilting the surface. The sections were transferred into the sublimation apparatus and dried for several minutes prior to matrix vapor deposition.

\section{Matrix vapor deposition}

Matrix deposition was carried out using an in-house built sublimation apparatus (for details, see Fig. S2 in the Electronic Supplementary Material, ESM). An ITO glass slide with an aortic tissue section was fixed onto the cooling plate using adhesive tape. Two hundred milligrams of 1,5-DAN was placed in a glass Petri dish on top of a hot plate. After closing the apparatus, the vacuum was kept below $2 \times 10^{-3}$ mbar using a rough pump and the hot plate was heated to a maximum temperature of $136{ }^{\circ} \mathrm{C}$. The sample was cooled below $20^{\circ} \mathrm{C}$, while sublimation was performed within 5 min at an increasing plate temperature between 123 and $136{ }^{\circ} \mathrm{C}$ and gave a DAN coating at around $0.11 \mathrm{mg} \times \mathrm{cm}^{-2}$ (see Fig. S2 in the ESM).

\section{Matrix recrystallization}

After matrix deposition, the sublimation apparatus was opened, the excessive matrix removed, and the hot plate quickly cooled down to about $20^{\circ} \mathrm{C}$ above the boiling point of the solvent used for recrystallization. A glass Petri dish was filled with $5 \mathrm{~mL}$ of acetonitrile, chloroform, or toluene and put on the hot plate. The apparatus was then closed for $30 \mathrm{~s}$ in order to expose the matrix coating of the sample to the vapor of the boiling solvent (see Fig. S2 in the ESM).

\section{MALDI MSI}

After sample preparation, ITO slides were locked in a Bruker MTP Slide Adapter II and immediately transferred into the vacuum system of an Ultraflex I MALDI TOF mass spectrometer equipped with a SmartBeam II Nd:YAG laser (Bruker Daltonik GmbH, Bremen, Germany). The laser spot size was set to $35 \mu \mathrm{m}$ [14], while the laser fluence was optimized to obtain the best signal-to-noise $(\mathrm{S} / \mathrm{N})$ ratio and kept constant within a set of experiments. The MS data were acquired in the range from $\mathrm{m} / \mathrm{z} 500$ to 1000 by averaging 50 mass spectra at every single spot. In general, samples were rastered in the positive ion-mode first and subsequently, with an offset of approx. $50 \mu \mathrm{m}$, in the negative ion-mode at a lateral resolution of $110 \times 110 \mu \mathrm{m}$. MSI raw data were processed utilizing the open source software mMass [24] as well as the MALDIquant (Version 1.16.2) [25] package and the Cardinal (Version 1.8.0) [26] package for the open-source $\mathrm{R}$ environment.

\section{Histological staining}

After MSI data acquisition, the matrix was removed using ethanol. A standard protocol was then used to stain the aortic slices with hematoxylin and eosin (H\&E) [27].

\section{LC-MS}

High-resolution mass spectrometric data were obtained by performing LC-MS and LC-MS/MS analyses of methanolic extracts of aortic tissue slices in the positive as well as negative ion-mode (for details, see ESM) [28]. Lipid identification was performed by comparing accurate mass data with the LIPID MAPS structure database [29] and further confirmed by tandem MS measurements.

\section{Results and discussion}

The thoracic aortic wall is composed of three different cell layers: the outer vascular wall (adventitia), the medial layer (media), and an inner monolayer (intima). Due to the small overall size of aortic cryosections of approx. 5-8 $\mathrm{mm}$ in length 
by $2 \mathrm{~mm}$ in width (see Figs. 1(a) and 2) and in order to exclude unwanted lateral analyte diffusion, we considered matrix vapor deposition/recrystallization $[11,18,19]$ to be the method of choice for the production of highly homogenous matrix coatings for high-resolution MSI. While coatings obtained from widely used matrices such as 2,5-dihydroxybenzoic acid or $\alpha$-cyano-4-hydroxycinnamic acid gave reasonable signal intensities for positive ions, these matrices turned out not to be feasible for the ionization of lipids in the negative mode [30].

According to literature, we obtained best results for imaging of lipids in aortic tissue in positive as well as negative ionmode by using 1,5-DAN [30-32]. We used two different setups for matrix vapor deposition depending on the size of the solid support for the tissue (see ESM, Figs. S1 and S2). In any case, we avoided the cryosections to be exposed to open air at ambient temperature for more than a few seconds (for a photographic image of the matrix coating, see Fig. 1(b)). Additionally, during matrix deposition in vacuo, the samples were continuously cooled. Without matrix recrystallization, average spectra of positive ion-mode MALDI MSI showed 55 lipid-related signals in the range of $\mathrm{m} / \mathrm{z} 650$ to 850 . We performed an automated peak picking with a signal-to-noise threshold $>6$, followed by an automated deisotoping and manually excluded signals showing non-typical mass defects
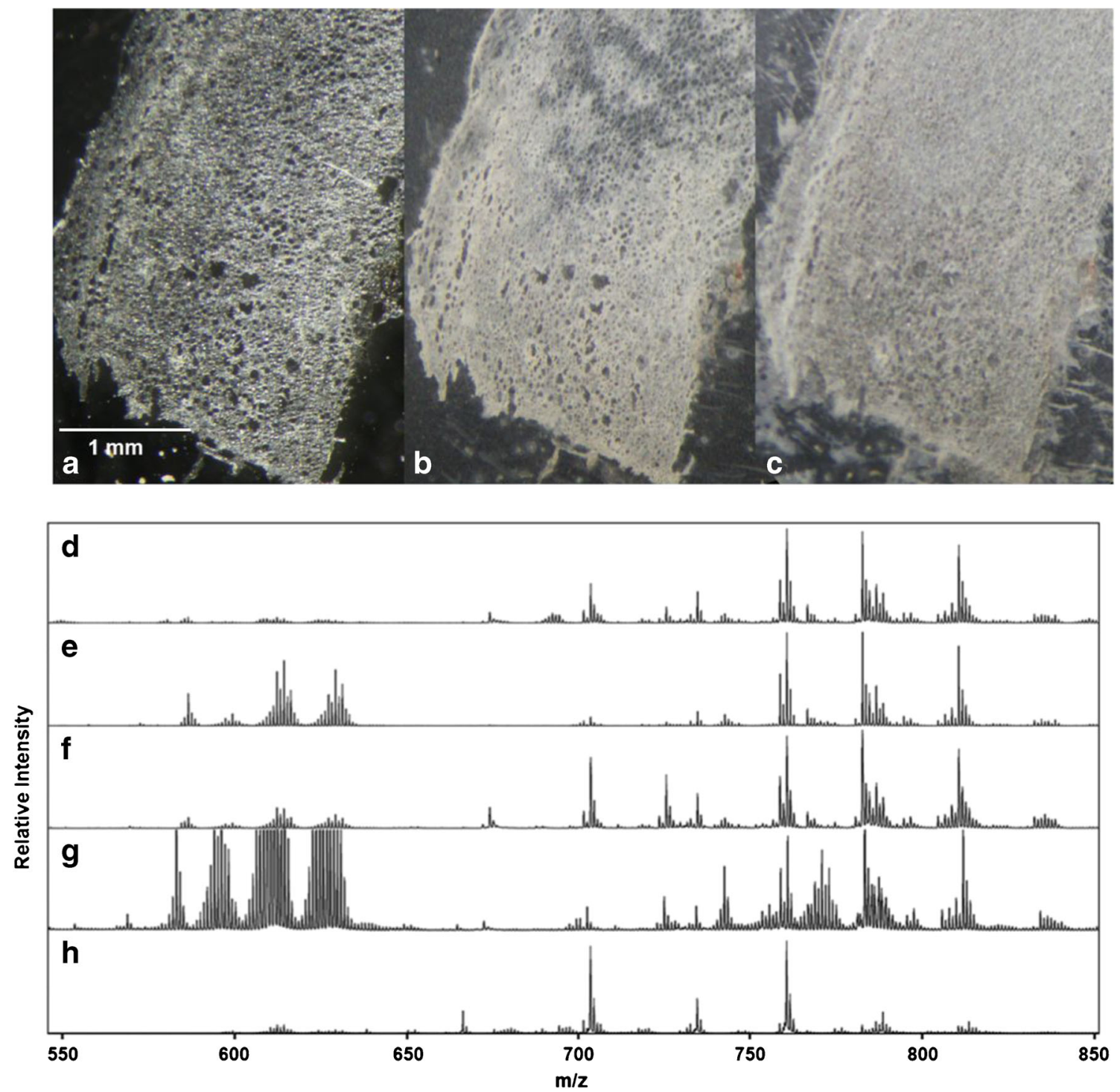

Fig. 1 (a-c) Microscopic images of aortic tissue: (a) untreated tissue section, (b) tissue section after DAN matrix vapor deposition, and (c) after matrix recrystallization using toluene. (d-h) Effect of sample preparation on the signal intensities of lipid-related signals in the positive ion-mode. Average mass spectra in the range of $\mathrm{m} / \mathrm{z} .550$ to 850 were obtained by rastering whole tissue sections: (d) DAN matrix vapor deposition and recrystallization using toluene, (e) DAN matrix vapor deposition without recrystallization, (f) aortic tissue washed with $\mathrm{NaCl}$ solution prior to DAN matrix vapor deposition and recrystallization with toluene, $(\mathbf{g})$ aortic tissue washed with $\mathrm{NaCl}$ solution prior to DAN matrix vapor deposition without recrystallization, (h) aortic tissue washed with $\mathrm{NH}_{4} \mathrm{HCO}_{2}$ solution prior to DAN matrix vapor deposition and recrystallization with toluene. For the negative mode, see the Electronic Supplementary Material 

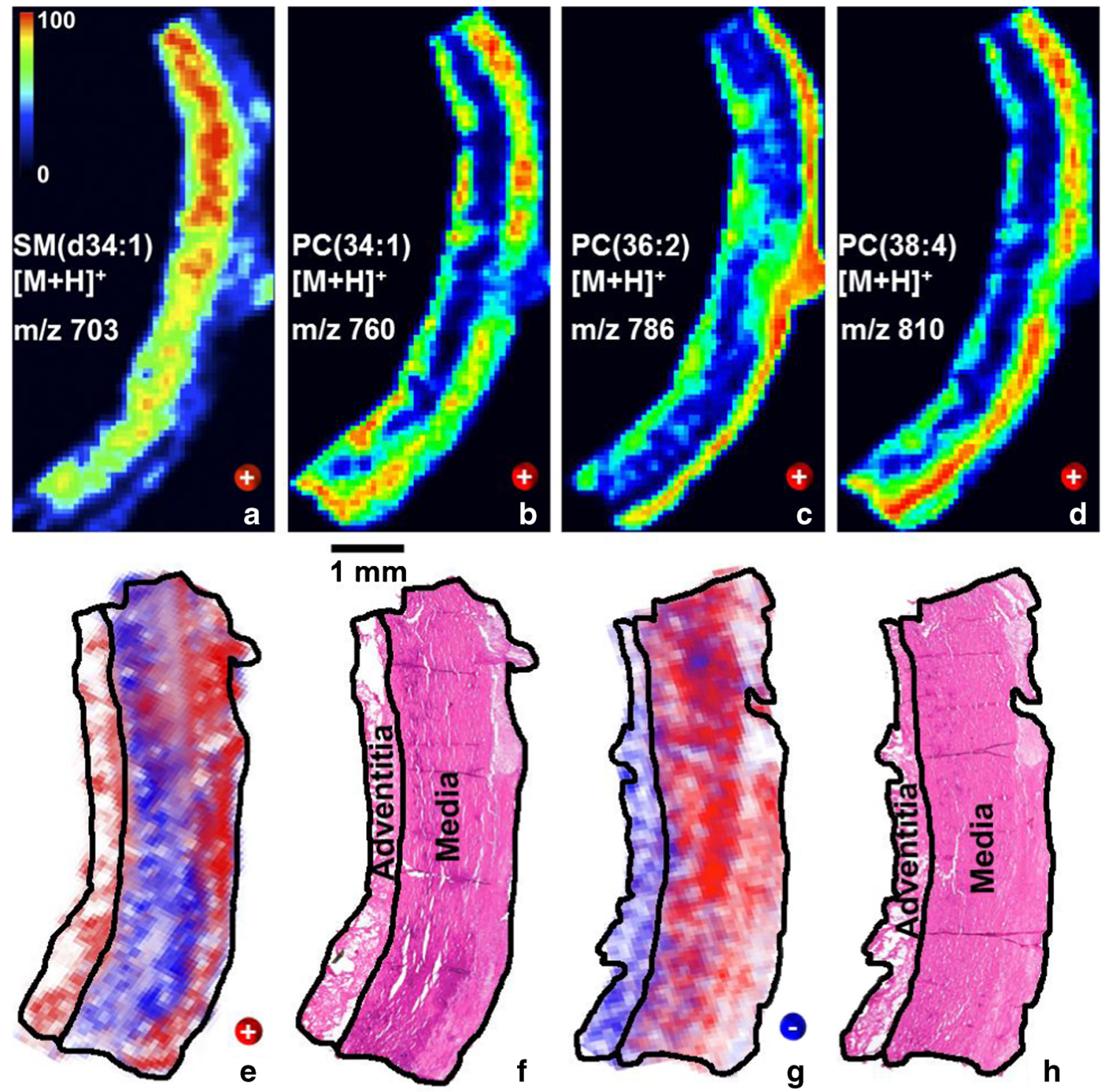

Fig. 2 MALDI-TOF MSI of lipids in human aortic tissue. Cryosections ( $8 \mu \mathrm{m}$ thick) were thaw-mounted on the ITO slides and coated with 1,5DAN. After matrix recrystallization using toluene, the samples were rastered at a lateral resolution of $110 \mu \mathrm{m}$ (laser spot size was set to $35 \mu \mathrm{m})$ : (a-d) Positive mode ion images of $\mathrm{SM}(\mathrm{d} 34: 1)$ at $\mathrm{m} / \mathrm{z} .703$ $\left([\mathrm{M}+\mathrm{H}]^{+}\right), \mathrm{PC}(34: 1)$ at $\mathrm{m} / z, 760\left([\mathrm{M}+\mathrm{H}]^{+}\right), \mathrm{PC}(36: 2)$ at $\mathrm{m} / z, 786([\mathrm{M}+$ $\left.\mathrm{H}]^{+}\right)$, and $\mathrm{PC}(38: 4)$ at $m / z 810\left([\mathrm{M}+\mathrm{H}]^{+}\right)$are plotted using a color scale

in a Kendrick mass defect plot [33-35]. However, in the range of $m / z 570$ to 650 , a not negligible set of interfering signals was observed. Kendrick mass defect plots as well as further experiments revealed these clusters to originate from the DAN matrix (Fig. 1(e); Fig. S3 and Table S1 in the ESM). In order to suppress matrix-related signals and further enhance the sensitivity of the method, the effect of a matrix recrystallization was investigated. We tested different non-polar solvents such as chloroform, acetonitrile, and toluene. After matrix vapor deposition the sublimation apparatus was filled with a few milliliters of solvent, and the matrix coating was exposed to the vapor of the solvent at ambient pressure for about 10 to $30 \mathrm{~s}$ (for a

from black (0\%) to red (100\%); (e) positive mode ion images of PC(36:2) at $m / z .786\left([\mathrm{M}+\mathrm{H}]^{+}\right.$; red) superimposed by $\mathrm{SM}(\mathrm{d} 34: 1)$ at $m / z .703([\mathrm{M}+$ $\mathrm{H}]^{+}$; blue) and the corresponding H\&E stained tissue (f); (g) negative mode ion images of $\mathrm{PC}(34: 4)$; at $\mathrm{m} / z .766\left(\left[\mathrm{M}-\mathrm{CH}_{3}\right]^{-}\right.$; blue $)$ superimposed by $\mathrm{SM}(\mathrm{d} 42: 1)$ at $\mathrm{m} / z .799\left(\left[\mathrm{M}-\mathrm{CH}_{3}\right]^{-}\right.$; red $)$and the corresponding H\&E stained aortic tissue (h)

photographic image of the recrystallized matrix coating on top of the aortic tissue, see Fig. 1(c)). The use of toluene as a solvent for recrystallization turned out to be most efficient. As depicted in Fig. 1(d), matrix cluster formation was found to be well suppressed while lipid-related signals stood out. A detailed comparison of the signal-to-noise ratios before and after recrystallization clearly indicated the advantages of a recrystallization step: under identical experimental conditions absolute signal intensities were found to be increased by $30-65 \%$ in the negative ion-mode, and from 5 to $23 \%$ in the positive ionmode (see ESM, Fig. S7). Polarized light microscopic images of DAN coating showed that a recrystallization step transforms 
the amorphous coating into a microcrystalline surface structure. The crystal size of recrystallized DAN is estimated to be in the low micrometer range (see Fig. S5 in the ESM). Furthermore, we used pheophytin, a non-polar, colored, and toluene soluble chlorophyll derivative, to study the lateral analyte diffusion during recrystallization. Analyte diffusion turned out to be above $20 \mu \mathrm{m}$ when the matrix was recrystallized for more than $30 \mathrm{~s}$ (see Fig. S6 of the ESM).

In order to control adduct formation in the positive ionmode, the effect of tissue washing prior to matrix deposition was investigated. The use of physiological saline solution as a sodium donor increased the total number of lipid-related signals to 67 , but did not reduce the abundance of protonated molecular ions. Moreover, matrix cluster formation turned out to be significantly enhanced, even after matrix recrystallization (Fig. 1(f, g)). On the other side, a tissue washing step using ammonium formate as a proton donor enhanced chemical noise throughout the spectrum and clearly reduced its signal-to-noise ratio. However, it successfully suppressed the formation of sodiated as well as potassiated molecular ions and turned out to be essential for signal assignment of the 48 lipid-related protonated molecular ions (Fig. 1(h) and ESM, Tables S1, S2, and S3).

Due to the lack of instrumental performance we obtained mass accuracies in the average mass spectra below $30 \mathrm{ppm}$. In order to avoid false assignments, we extracted two 8 - $\mu \mathrm{m}$ tissue sections (adjacent to the imaged section) and performed LC-MS and LC-MS/MS experiments in positive and negative ion-mode. The obtained high-resolution data (see Electronic Supplementary Material) enabled the unambiguous determination of the elemental compositions of overall 55 different lipids and thus an internal recalibration of our TOF data. Moreover, our aortic tissue samples originate from a study done by Doppler et al. in 2017 where the authors published the identification and quantification of dozens of lipids using flow injection analysis (FIA) MS/MS [22]. Hence, based on three different sources of lipidomics data and considering standards of lipid identification and annotation [36], we were able to assign 34 lipid-related signals obtained from positive as well as negative ion-mode MALDI-TOF MSI (see ESM, Tables S2 and S3, Figs. S9A and S9B).

In conclusion, best results in terms of a significant enhancement of lipid-related signals (up to $65 \%$ in negative as well as up to $23 \%$ in positive ion-mode) for the detection of a maximum number of lipid-related signals in aortic tissue were obtained when untreated, thaw-mounted cryosections were coated with 1,5-DAN and toluene was used for recrystallization (see Fig. 2 and Figs. S3, S4, and S7 in the ESM). When an offset of a few micrometers is applied, the sample can consecutively be analyzed in the positive as well as negative ionmode (ESM Fig. S9) [27, 30]. For lipid identification, a tissue washing using $\mathrm{NH}_{4} \mathrm{HCO}_{2}$ solution is recommended, which enforces the formation of protonated molecular ions.
As can be seen in Fig. 2, the tunica media layer of the aortic blood vessel is, according to H\&E staining ( $2 \mathrm{f}$ and $2 \mathrm{~h}$ ), a uniform layer of cells. On the contrary, imaging mass spectrometry clearly reveals that on a molecular level, the media is by far not homogenous and features several so far unknown lateral distributions of different lipid species. Since lipids play essential roles in diverse tissues as constituents of cell membranes, act as regulators for various biological processes, and are associated with many diseases in which lipids show a modified distribution in tissue [37-40], our observations call for further investigations in order to clarify a possible physiological importance of lipid distribution in case of TAA.

In order to detect and identify even low abundant species using high-resolution MALDI MSI, a robust and fast sample preparation [36] featuring high $\mathrm{S} / \mathrm{N}$ ratio as well as low chemical noise is essential. The presented method comprising an optional tissue washing followed by matrix vapor deposition/ recrystallization provides a sample preparation within minutes and will easily be adapted for an application on different types of biologically relevant tissues.

Acknowledgements Open access funding provided by University of Innsbruck and Medical University of Innsbruck. We would like to thank Prof. Roland Stalder from the Institute of Mineralogy and Petrography at the University of Innsbruck for providing polarized light microscopy images. Further, we want to thank Dr. Silye from the Department of Pathology, Linz General Hospital, Johannes Kepler University, Linz, Austria, for scanning of the histological tissue slides. Financial support by the Bundesministerium für Bildung, Wissenschaft und Forschung (BMBWF, Project SPA/04-140/Indian Summer in Tyrol to TM), the University of Innsbruck (scholarship to $\mathrm{CM}$ ) and the Tiroler Wissenschaftsfonds (TWF-2016-1-9 to CD) is gratefully acknowledged.

\section{Compliance with ethical standards}

The study conforms to the Declaration of Helsinki and has been approved by the Ethics Committee of the Medical University of Innsbruck and the Ethics Committee of the Medical University of Vienna. Written informed consent from all study participants is available.

Conflict of interest The authors declare that they have no conflict of interest.

Open Access This article is distributed under the terms of the Creative Commons Attribution 4.0 International License (http:// creativecommons.org/licenses/by/4.0/), which permits unrestricted use, distribution, and reproduction in any medium, provided you give appropriate credit to the original author(s) and the source, provide a link to the Creative Commons license, and indicate if changes were made.

\section{References}

1. Caprioli RM, Farmer TB, Gile J. Molecular imaging of biological samples: localization of peptides and proteins using MALDI-TOF MS. Anal Chem. 1997;69(23):4751-60. https://doi.org/10.1021/ ac970888i. 
2. Stoeckli M, Chaurand P, Hallahan DE, Caprioli RM. Imaging mass spectrometry: a new technology for the analysis of protein expression in mammalian tissues. Nat Med. 2001;7(4):493-6. https://doi. org/10.1038/86573.

3. Cornett DS, Reyzer ML, Chaurand P, Caprioli RM. MALDI imaging mass spectrometry: molecular snapshots of biochemical systems. Nat Methods. 2007;4(10):828-33. https://doi.org/10.1038/ Nmeth1094.

4. Chaurand P, Norris JL, Cornett DS, Mobley JA, Caprioli RM. New developments in profiling and imaging of proteins from tissue sections by MALDI mass spectrometry. J Proteome Res. 2006;5(11): 2889-900. https://doi.org/10.1021/pr060346u.

5. Juhasz P, Costello CE, Biemann K. Matrix-assisted laser desorption ionization mass-spectrometry with 2-(4-hydroxyphenylazo)benzoic acid matrix. J Am Soc Mass Spectrom. 1993;4(5):399-409. https:// doi.org/10.1016/1044-0305(93)85005-I.

6. Axelsson J, Hoberg AM, Waterson C, Myatt P, Shield GL, Varney $\mathrm{J}$, et al. Improved reproducibility and increased signal intensity in matrix-assisted laser desorption/ionization as a result of electrospray sample preparation. Rapid Commun Mass Spectrom. 1997;11(2):209-13. https://doi.org/10.1002/(Sici)10970231(19970131)11:2<209::Aid-Rcm734>3.0.Co;2-E.

7. Schwartz SA, Reyzer ML, Caprioli RM. Direct tissue analysis using matrix-assisted laser desorption/ionization mass spectrometry: practical aspects of sample preparation. J Mass Spectrom. 2003;38(7):699-708. https://doi.org/10.1002/jms.505.

8. Baluya DL, Garrett TJ, Yost RA. Automated MALDI matrix deposition method with inkjet printing for imaging mass spectrometry. Anal Chem. 2007;79(17):6862-7. https://doi.org/10.1021/ ac070958d.

9. Wang HYJ, Post SNJJ, Woods AS. A minimalist approach to MALDI imaging of glycerophospholipids and sphingolipids in rat brain sections. Int J Mass Spectrom. 2008;278(2-3):143-9. https:// doi.org/10.1016/j.ijms.2008.04.005.

10. Chen YF, Allegood J, Liu Y, Wang E, Cachon-Gonzalez B, Cox $\mathrm{TM}$, et al. Imaging MALDI mass spectrometry using an oscillating capillary nebulizer matrix coating system and its application to analysis of lipids in brain from a mouse model of Tay-Sachs/ Sandhoff disease. Anal Chem. 2008;80(8):2780-8. https://doi.org/ 10.1021/ac702350g.

11. Hankin JA, Barkley RM, Murphy RC. Sublimation as a method of matrix application for mass spectrometric imaging. J Am Soc Mass Spectrom. 2007;18(9):1646-52. https://doi.org/10.1016/j.jasms. 2007.06.010.

12. Puolitaival SM, Burnum KE, Cornett DS, Caprioli RM. Solventfree matrix dry-coating for MALDI imaging of phospholipids. J Am Soc Mass Spectrom. 2008;19(6):882-6. https://doi.org/10. 1016/j.jasms.2008.02.013.

13. Yang JY, Phelan VV, Simkovsky R, Watrous JD, Trial RM, Fleming TC, et al. Primer on agar-based microbial imaging mass spectrometry. J Bacteriol. 2012;194(22):6023-8. https://doi.org/10.1128/Jb. 00823-12.

14. Vergeiner S, Schafferer L, Haas H, Muller T. Improved MALDITOF microbial mass spectrometry imaging by application of a dispersed solid matrix. J Am Soc Mass Spectrom. 2014;25(8):1498501. https://doi.org/10.1007/s13361-014-0923-y.

15. Caughlin S, Park DH, Yeung KKC, Cechetto DF, Whitehead SN. Sublimation of DAN matrix for the detection and visualization of gangliosides in rat brain tissue for MALDI imaging mass spectrometry. J Vis Exp. 2017;(121). ARTN e55254). https://doi.org/10. $3791 / 55254$.

16. Aerni HR, Cornett DS, Caprioli RM. Automated acoustic matrix deposition for MALDI sample preparation. Anal Chem. 2006;78(3):827-34. https://doi.org/10.1021/ac051534r.

17. Jaskolla TW, Karas M, Roth U, Steinert K, Menzel C, Reihs K. Comparison between vacuum sublimed matrices and conventional dried droplet preparation in MALDI-TOF mass spectrometry. J Am Soc Mass Spectrom. 2009;20(6):1104-14. https://doi.org/10.1016/ j.jasms.2009.02.010.

18. Bouschen W, Schulz O, Eikel D, Spengler B. Matrix vapor deposition/recrystallization and dedicated spray preparation for high-resolution scanning microprobe matrix-assisted laser desorption/ionization imaging mass spectrometry (SMALDI-MS) of tissue and single cells. Rapid Commun Mass Spectrom. 2010;24(3):355-64. https://doi.org/10.1002/rcm.4401.

19. Yang JH, Caprioli RM. Matrix sublimation/recrystallization for imaging proteins by mass spectrometry at high spatial resolution. Anal Chem. 2011;83(14):5728-34. https://doi.org/10.1021/ac200998a.

20. Prevention NCfDCa. Wisqars leading causes of death reports, 1999-2016; 2018.

21. Elefteriades JA, Farkas EA. Thoracic aortic aneurysm clinically pertinent controversies and uncertainties. J Am Coll Cardiol. 2010;55(9):841-57. https://doi.org/10.1016/j.jacc.2009.08.084.

22. Doppler C, Arnhard K, Dumfarth J, Heinz K, Messner B, Stern C, et al. Metabolomic profiling of ascending thoracic aortic aneurysms and dissections - implications for pathophysiology and biomarker discovery. PLoS One. 2017;12(5):e0176727. https://doi.org/10. 1371/journal.pone.0176727.

23. Angel PM, Spraggins JM, Baldwin HS, Caprioli R. Enhanced sensitivity for high spatial resolution lipid analysis by negative ion mode matrix assisted laser desorption ionization imaging mass spectrometry. Anal Chem. 2012;84(3):1557-64. https://doi.org/10. $1021 / \mathrm{ac} 202383 \mathrm{~m}$

24. Strohalm M, Hassman M, Kosata B, Kodicek M. mMass data miner: an open source alternative for mass spectrometric data analysis. Rapid Commun Mass Spectrom. 2008;22(6):905-8. https://doi.org/ 10.1002/rcm.3444.

25. Gibb S, Strimmer K. MALDIquant: a versatile R package for the analysis of mass spectrometry data. Bioinformatics. 2012;28(17): 2270-1. https://doi.org/10.1093/bioinformatics/bts447.

26. Bemis KD, Harry A, Eberlin LS, Ferreira C, van de Ven SM, Mallick P, et al. Cardinal: an R package for statistical analysis of mass spectrometry-based imaging experiments. Bioinformatics. 2015;31(14):2418-20. https://doi.org/10.1093/bioinformatics/ btv146.

27. Thomas A, Patterson NH, Marcinkiewicz MM, Lazaris A, Metrakos P, Chaurand P. Histology-driven data mining of lipid signatures from multiple imaging mass spectrometry analyses: application to human colorectal cancer liver metastasis biopsies. Anal Chem. 2013;85(5):2860-6. https://doi.org/10.1021/ac3034294.

28. Sato Y, Bernier F, Suzuki I, Kotani S, Nakagawa M, Oda Y. Comparative lipidomics of mouse brain exposed to enriched environment. J Lipid Res. 2013;54(10):2687-96. https://doi.org/10. 1194/jlr.M038075.

29. https://www.lipidmaps.org

30. Thomas A, Charbonneau JL, Fournaise E, Chaurand P. Sublimation of new matrix candidates for high spatial resolution imaging mass spectrometry of lipids: enhanced information in both positive and negative polarities after 1,5diaminonapthalene deposition. Anal Chem. 2012;84(4):204854. https://doi.org/10.1021/ac2033547.

31. Dong W, Shen Q, Baibado JT, Liang YM, Wang P, Huang YQ, et al. Phospholipid analyses by MALDI-TOF/TOF mass spectrometry using 1,5-diaminonaphthalene as matrix. Int J Mass Spectrom. 2013;343:15-22. https://doi.org/10.1016/j.ijms.2013.04.004.

32. Yang E, Dufresne M, Chaurand P. Enhancing ganglioside species detection for MALDI-TOF imaging mass spectrometry in negative reflectron mode. Int J Mass Spectrom. 2019;437:3-9. https://doi. org/10.1016/j.ijms.2017.09.011.

33. Kendrick E. A mass scale based on $\mathrm{Ch} 2=14.0000$ for high resolution mass spectrometry of organic compounds. Anal Chem. 1963;35(13):2146. https://doi.org/10.1021/ac60206a048. 
34. Hughey CA, Hendrickson CL, Rodgers RP, Marshall AG, Qian KN. Kendrick mass defect spectrum: a compact visual analysis for ultrahigh-resolution broadband mass spectra. Anal Chem. 2001;73(19):4676-81. https://doi.org/10.1021/ ac010560w.

35. Lerno LA, German JB, Lebrilla CB. Method for the identification of lipid classes based on referenced Kendrick mass analysis. Anal Chem. 2010;82(10):4236-45. https://doi.org/10.1021/ac100556g.

36. Liebisch G, Ekroos K, Hermansson M, Ejsing CS. Reporting of lipidomics data should be standardized. Biochim Biophys Acta Mol Cell Biol Lipids. 2017;1862(8):747-51. https://doi.org/10. 1016/j.bbalip.2017.02.013.

37. Dowhan W. Molecular basis for membrane phospholipid diversity: why are there so many lipids? Annu Rev Biochem. 1997;66:199232. https://doi.org/10.1146/annurev.biochem.66.1.199.
38. Murphy RC, Hankin JA, Barkley RM. Imaging of lipid species by MALDI mass spectrometry. J Lipid Res. 2009;50:S317-S22. https://doi.org/10.1194/jlr.R800051-JLR200.

39. Li M, Yang L, Bai Y, Liu H. Analytical methods in lipidomics and their applications. Anal Chem. 2014;86(1):161-75. https://doi.org/ 10.1021/ac403554h.

40. Tanaka H, Zaima N, Sasaki T, Yamamoto N, Inuzuka K, Sano M, et al. Imaging mass spectrometry reveals a unique distribution of triglycerides in the abdominal aortic aneurysmal wall. J Vasc Res. 2015;52(2):127-35. https://doi.org/10.1159/000439169.

Publisher's note Springer Nature remains neutral with regard to jurisdictional claims in published maps and institutional affiliations. 